\title{
Distributed Stochastic MPC of Linear Systems with Additive Uncertainty and Coupled Probabilistic Constraints
}

\author{
Li Dai, Yuanqing Xia, Yulong Gao, and Mark Cannon
}

\begin{abstract}
This paper develops a new form of distributed stochastic model predictive control (DSMPC) algorithm for a group of linear stochastic subsystems subject to additive uncertainty and coupled probabilistic constraints. We provide an appropriate way to design the DSMPC algorithm by extending a centralized SMPC (CSMPC) scheme. To achieve the satisfaction of coupled probabilistic constraints in a distributed manner, only one subsystem is permitted to optimize at each time step. In addition, by making explicit use of the probabilistic distribution of the uncertainties, probabilistic constraints are converted into a set of deterministic constraints for the predictions of nominal models. The distributed controller can achieve recursive feasibility and ensure closed-loop stability for any choice of update sequence. Numerical examples illustrate the efficacy of the algorithm.
\end{abstract}

Index Terms-Distributed control, model predictive control (MPC), probabilistic constraints, stochastic systems.

\section{INTRODUCTION}

I $\mathrm{N}$ recent years, stochastic model predictive control (SMPC) has attracted much attention since it is an alternative MPC scheme to deal with uncertain systems. Different from robust MPC (RMPC), SMPC takes into account the stochastic characteristics of the uncertainties and allows constraint violations to occur with a frequency below a prespecified threshold, i.e. probabilistic constraints. Probabilistic constraints enable SMPC to directly incorporate the tradeoffs between the satisfaction of constraints and control performance, and therefore alleviate the inherent conservatism of RMPC [1]-[4].

Distributed MPC (DMPC) is a further development of MPC technique for large-scale systems, such as chemical plants [5], process control [6], or teams of vehicles [7]. Compared with centralized MPC scheme, DMPC is more attractive in the context of computation and communication. For large-scale systems with the uncertainties, it is natural to develop RMPC or SMPC to the distributed framework, namely distributed

Manuscript received XXXX, XXXX; revised XXXX, XXXX; accepted XXXX, XXXX. Date of publication XXXX, XXXX; date of current version XXXX, XXXX. This work was supported by the National Basic Research Program of China (973 Program) under Grant 2012CB720000, the National Natural Science Foundation of China under Grant 61603041, Grant 61225015 , Grant 61105092 and Grant 61422102, the Beijing Natural Science Foundation under Grant 4161001, and by Foundation for Innovative Research Groups of the National Natural Science Foundation of China under Grant 61321002.

Li Dai and Yuanqing Xia are with School of Automation, Beijing Institute of Technology, Beijing 100081, China (e-mail: daili1887@gmail.com; xia_yuanqing@bit.edu.cn). Yulong Gao is with ACCESS Linnaeus Center, KTH Royal Institute of Technology, SE-10044 Stockholm, Sweden (e-mail: dfgaoyulong@gmail.com). Mark Cannon is with Department of Engineering Science, University of Oxford, Parks Road, Oxford, OX1 3PJ, United Kingdom (e-mail: mark.cannon@eng.ox.ac.uk).
RMPC (DRMPC) or distributed SMPC (DSMPC). This paper aims to establish some fundamental results on DSMPC by promoting the current SMPC technique to the distributed case in a more systematical way. These fundamental results include the key points in SMPC, such as the treatment of probabilistic constraints, recursive feasibility, and closed-loop stability. Next we will compare the existing SMPC algorithms for a single system and inherit some ideas of them to develop our DSMPC algorithm by proposing some distributed techniques.

Nowadays, three representative approaches for SMPC of a single system include stochastic tube approaches [8], [9], approaches based on affine parameterization of the control policy [10], [11], and sample-based approaches [12], [13]. From the perspective of computational complexity and the establishment of theoretical results, stochastic tube approaches are more possibly scalable to the distributed manner than other two approaches. Particularly, the recent work [9] adopted the stochastic tube methodology to address the probabilistic constraints and ensured recursive feasibility and closed-loop stability. A sufficient and necessary condition was proposed to ensure the satisfaction of probabilistic constraints, thereby incurring no additional conservatism. However, the results of [9] were restricted to the case of a single system. In the presence of coupling among several subsystems, additional techniques are needed to promote the results of [9] to a distributed case while guaranteeing recursive feasibility and closed-loop stability of the whole systems.

The handling of the coupling in DMPC mainly depends on the choice of update strategy. In this paper, we will formulate the coupling among subsystems in a general form of coupled probabilistic constraints. Some approaches in DRMPC have been investigated to ensure that the distributed actions satisfy coupled constraints, see [14]-[16]. One of these approaches is the sequential solution scheme adopted in [16], which required to optimize all the subsystems sequentially at one time step. Using the similar method to [16], the satisfaction of coupled constraints was guaranteed in [17] by local subsystems updating plans one at a time, without iteration. In [17], the subsystems can be updated in any choice of update sequence rather than in a prescribed sequence as in [18]. Under these update strategies, all the aforementioned DRMPC can ensure recursive feasibility and closed-loop stability. Compared with DRMPC, few DSMPC methods are available. Particularly, when subsystems share coupled probabilistic constraints, the existing methods (e.g., [19]) cannot ensure recursive feasibility and closed-loop stability. 
Motivated by above discussion, this paper proposes a distributed form of the recently developed SMPC in [9] for a team of linear stochastic subsystems with independent dynamics but coupled probabilistic constraints. We mainly use the idea of [17] that, at each time step, a single subsystem is permitted to optimize, while other subsystems 'freeze' their plans. Apart from this, the controller structure and the stochastic disturbances considered here are significantly different from those of [17]. From the perspective of the SMPC theory, the contributions of this paper are listed below. First, by making explicit use of the probabilistic distribution of the uncertainties and using a distributed way, deterministic constraints are constructed to provide coupled probabilistic constraint satisfaction non-conservatively, in the sense that the specified violation value can be reached tightly. Second, under the assumption that the additive uncertainty is finitely supported, the initial feasibility of the optimization problem implies the feasibility at all times. Third, closed-loop stability of the overall system is guaranteed for any choice of update sequence. Finally, two different aspects between this paper and our recent work [20] need clarification. Compared with the results of this paper, (i) the cooperation scheme in [20] brought in a better global control performance but resulted in higher computation and communication; (ii) the closed-loop stability of [20] depended on an additional assumption (Assumption 1 in [20]).

The outline of this paper is as follows. Section II presents the problem statement for a team of subsystems with coupled probabilistic constraints. Section III reviews SMPC by applying it in a centralized fashion to the problem of Section II. Section IV develops a DSMPC algorithm, which can ensure recursive feasibility and stability. Section $\mathrm{V}$ presents the results from numerical simulations and compares the global performance of the new algorithm with that of a related DRMPC algorithm. Finally, conclusions are drawn in Section VI.

Notation: Let $\mathbb{N} \triangleq\{0,1,2, \ldots\}$; for a symmetric matrix $X$, $X \succ 0$ and $X \succeq 0$ mean that $X$ is a positive definite matrix and a positive-semidefinite matrix, respectively; for a vector $x, x_{i}$ is the $i$ th component of $x$; the inequality sign $\leq$ and the absolute value $|\cdot|$ apply elementwise to vectors; $\|x\|_{W}^{2}=$ $x^{T} W x ;(k+i \mid k)$ indicates a prediction of a variable $i$ steps ahead from time $k ; \operatorname{Pr}$ is the probability, $\mathbb{E}$ is the expectation, and $I$ is the identity matrix with proper dimension.

\section{PRoBlem STATEMENT}

Consider a system of $N_{p}$ linear time-invariant, discrete-time subsystems which are assumed to be stabilizable,

$$
\begin{gathered}
x_{p}(k+1)=A_{p} x_{p}(k)+B_{p} u_{p}(k)+G_{p} w_{p}(k), \\
\forall k \in \mathbb{N}, \quad \forall p \in \mathcal{P} \triangleq\left\{1,2, \ldots, N_{p}\right\},
\end{gathered}
$$

where $x_{p} \in \mathbb{R}^{N_{x, p}}, u_{p} \in \mathbb{R}^{N_{u, p}}$, and $w_{p} \in \mathbb{R}^{N_{w, p}}$ are the state, the control input, and the disturbance acting on subsystem $p$, respectively. It is assumed that the complete state $x_{p}(k)$ is available at each time $k$. Assume that $\left\{w_{p}(0), w_{p}(1), \ldots\right\}$ is independent and identically distributed (i.i.d.) for each subsystem $p$, that the elements of $w_{p}(k)$ have mean zero, and that the distribution of the $i$ th element of $w_{p}(k)$ has the form

$$
\begin{gathered}
\operatorname{Pr}\left\{w_{p, i}(k) \leq \eta_{p, i}\right\}=F_{p, i}\left(\eta_{p, i}\right), \\
F_{p, i}\left(\eta_{p, i}\right)=\left\{\begin{array}{ll}
1 & \text { for } \quad \eta_{p, i} \geq \alpha_{p, i} \\
0 & \text { for } \quad \eta_{p, i}<-\alpha_{p, i}
\end{array},\right.
\end{gathered}
$$

where $\alpha_{p, i}>0$ and the distribution functions $F_{p, i}$ are assumed to be continuous. A consequence of (2) is that $w_{p}(k)$ is assumed to lie in the orthotope $w_{p}(k) \in W_{p} \triangleq\left\{w_{p}|| w_{p} \mid \leq\right.$ $\left.\alpha_{p}\right\}, \alpha_{p}=\left[\begin{array}{llll}\alpha_{p, 1} & \alpha_{p, 2} & \cdots & \alpha_{p, N_{w, p}}\end{array}\right]^{T}$.

Remark 2.1: Since the disturbances derived from physical processes are finite, the assumption of finitely supported uncertainties matches the real world more closely than the practically unrealistic Gaussian assumption. And this assumption is necessary to assert recursive feasibility and stability.

Each subsystem is assumed to be subject to one local probabilistic constraint on an output $y_{p}(k)$

$$
\begin{gathered}
y_{p}(k)=C_{p} x_{p}(k)+D_{p} u_{p}(k), y_{p}(k) \in \mathbb{R}, \\
\operatorname{Pr}\left\{y_{p}(k) \leq h_{p}\right\} \geq l_{p}, \quad \forall k \in \mathbb{N}, \forall p \in \mathcal{P} .
\end{gathered}
$$

There are also $N_{c}$ coupled probabilistic constraints across multiple subsystems, each of which applies to the sum of the coupled output $s_{c p}, c \in \mathcal{C} \triangleq\left\{1,2, \ldots, N_{c}\right\}$, from each subsystem

$$
\begin{aligned}
& s_{c p}(k)=E_{c p} x_{p}(k)+F_{c p} u_{p}(k), s_{c p}(k) \in \mathbb{R}, \\
& \operatorname{Pr}\left\{\sum_{p=1}^{N_{p}} s_{c p}(k) \leq b_{c}\right\} \geq p_{c}, \forall k \in \mathbb{N}, \forall c \in \mathcal{C} .
\end{aligned}
$$

The row vectors $C_{p}, D_{p}, E_{c p}, F_{c p}$, fixed scalars $h_{p}, b_{c}$, and thresholds $l_{p} \in[0,1], p_{c} \in[0,1]$ are all chosen by the designer. The form of (6) is very general, which permits coupling to exist between any number of subsystems. The case of several local probabilistic constraints of one subsystem can be treated similarly by deriving the online constraint for each individual constraint. And the case of hard constraints is treated simply by setting $l_{p}=1$ and $p_{c}=1$ in (4) and (6).

The predicted control input at time $k$ is made up of a linear state feedback and a perturbation

$$
u_{p}(k+i \mid k)=K_{p} x_{p}(k+i \mid k)+c_{p}(k+i \mid k),
$$

where $K_{p}$ is calculated offline such that $A_{p}+B_{p} K_{p}$ is strictly stable for each subsystem $\left(A_{p}, B_{p}\right)$. Given a finite prediction horizon $N, c_{p}(k+i \mid k) \in \mathbb{R}^{N_{u, p}}, i=0,1, \ldots, N-1$, are optimization variables and $c_{p}(k+i \mid k)=\mathbf{0}$ for $i \geq N$. Then we decompose the state trajectories predicted at time $k$ into two components: the nominal one $z_{p}(k+i \mid k)=\mathbb{E}\left[x_{p}(k+i \mid k)\right]$ and the uncertain one $e_{p}(k+i \mid k)$, which are formulated as

$$
\begin{gathered}
x_{p}(k+i \mid k)=z_{p}(k+i \mid k)+e_{p}(k+i \mid k), \\
z_{p}(k+i+1 \mid k)=\Phi_{p} z_{p}(k+i \mid k)+B_{p} c_{p}(k+i \mid k), \\
e_{p}(k+i+1 \mid k)=\Phi_{p} e_{p}(k+i \mid k)+G_{p} w_{p}(k+i \mid k),
\end{gathered}
$$

where $\Phi_{p}=A_{p}+B_{p} K_{p}$ and initial conditions pertaining to $i=0$ are $z_{p}(k \mid k)=x_{p}(k)$ and $e_{p}(k \mid k)=0$ (since the state at time $k$ is known by assumption).

To facilitate the illustration of the coupling structure between subsystems, as in [17], define $\mathcal{P}_{c} \triangleq\{p \in \mathcal{P} \mid$ $\left.\left[E_{c p} F_{c p}\right] \neq \mathbf{0}\right\}$ as the set of subsystems involved in coupled 
constraint $c$ and $\mathcal{C}_{p} \triangleq\left\{c \in \mathcal{C} \mid\left[E_{c p} F_{c p}\right] \neq \mathbf{0}\right\}$ as the set of coupled constraints involved in subsystem $p$. Then define $\mathcal{Q}_{p} \triangleq\left(\bigcup_{c \in \mathcal{C}_{p}} \mathcal{P}_{c}\right) \backslash\{p\}$ as the set of all other subsystems coupled to subsystem $p$. The following standing assumption shall be applied to the subsequent analysis.

Assumption 2.1: Each subsystem $p$ has a priori knowledge of static model parameters for subsystems $q \in \mathcal{Q}_{p}$. These will be used later to enable each subsystem $p$ to construct outputs of subsystems $q \in \mathcal{Q}_{p}$ given the states $x_{q}(k)$ and the sequences of perturbations.

\section{REVIEW OF CENTRALIZED SMPC}

This section reviews SMPC in [9] with application to the problem of Section II. To devise a centralized SMPC (CSMPC) strategy, at time $k$, minimize the cost function of the whole system

$$
\begin{aligned}
J_{k}(\mathbf{C}(k))=\sum_{p=1}^{N_{p}} \sum_{i=0}^{\infty} \mathbb{E}\left[x_{p}^{T}(k+i \mid k) Q x_{p}(k+i \mid k)\right. \\
\left.+u_{p}^{T}(k+i \mid k) R u_{p}(k+i \mid k)-L_{p}\right],
\end{aligned}
$$

subject to the constraints (4) and (6), where

$$
\begin{aligned}
L_{p}=\lim _{i \rightarrow \infty} \mathbb{E}[ & x_{p}^{T}(k+i \mid k) Q x_{p}(k+i \mid k) \\
& \left.+u_{p}^{T}(k+i \mid k) R u_{p}(k+i \mid k)\right]
\end{aligned}
$$

and $Q \succ 0$ and $R \succ 0$ are two known weighting matrices. The decision variable $\mathbf{C}(k) \triangleq\left[\mathbf{c}_{1}^{T}(k) \mathbf{c}_{2}^{T}(k) \cdots \mathbf{c}_{N_{p}}^{T}(k)\right]^{T}$ with $\mathbf{c}_{p}(k)=\left[c_{p}^{T}(k \mid k) c_{p}^{T}(k+1 \mid k) \cdots c_{p}^{T}(k+N-1 \mid k)\right]^{T^{p}} \in \mathbb{R}^{N_{u, p} N}$ for any $p \in \mathcal{P}$.

Remark 3.1: Due to the existence of the additive uncertainty in (1), the stage cost $\mathbb{E}\left[x_{p}^{T}(k+i \mid k) Q x_{p}(k+i \mid k)+\right.$ $\left.u_{p}^{T}(k+i \mid k) R u_{p}(k+i \mid k)\right]$ converges to a finite, non-zero limit $L_{p}$ so that the summation of each stage cost is infinite over the infinite prediction horizon. To obtain a finite cost, we define the cost function (11) in terms of the deviation of the stage cost from the limit value $L_{p}$, which can be precomputed using a similar method as in [21].

To ensure the satisfaction of (4) and (6) in closed-loop operation, the first step is to guarantee that the constraints are satisfied over the entire prediction horizon at time $k$ for all subsystems. The following theorem provides necessary and sufficient conditions for this.

Theorem 3.1: [9] At time $k$, probabilistic constraints (4) and (6) are satisfied by predictions of (1) for each subsystem $p \in \mathcal{P}$ if and only if for $i=1,2, \ldots, \mathbf{c}_{p}(k)$ satisfies

$$
\begin{aligned}
& \Psi_{p} \Phi_{p}^{i} z_{p}(k \mid k)+\left(\Psi_{p} H_{p}^{i}+D_{p} E_{p}^{i+1}\right) \mathbf{c}_{p}(k) \\
\leq & h_{p}-\gamma_{p}^{i}, \forall p \in \mathcal{P}, \\
& \sum_{p=1}^{N_{p}} \Delta_{c p} \Phi_{p}^{i} z_{p}(k \mid k)+\sum_{p=1}^{N_{p}}\left(\Delta_{c p} H_{p}^{i}+F_{c p} E_{p}^{i+1}\right) \mathbf{c}_{p}(k) \\
\leq & b_{c}-\xi_{c}^{i}, \forall c \in \mathcal{C},
\end{aligned}
$$

where $\Psi_{p}=C_{p}+D_{p} K_{p}, \Delta_{c p}=E_{c p}+F_{c p} K_{p}, H_{p}^{i}=$ $\left[\Phi_{p}^{i-1} B_{p} \cdots B_{p} \mathbf{0} \cdots \mathbf{0}\right], E_{p}^{i+1} \mathbf{c}_{p}(k)=c_{p}(k+i \mid k)$, and $\gamma_{p}^{i}$ and $\xi_{c}^{i}$ are respectively defined as the minimum values such that $\operatorname{Pr}\left\{\Psi_{p}\left[\Phi_{p}^{i-1} G_{p} w_{p}(k \mid k)+\ldots+G_{p} w_{p}(k+i-1 \mid k)\right] \leq \gamma_{p}^{i}\right\}=l_{p}$ and $\operatorname{Pr}\left\{\sum_{p=1}^{N_{p}} \Delta_{c p}\left[\Phi_{p}^{i-1} G_{p} w_{p}(k \mid k)+\ldots+G_{p} w_{p}(k+i-\right.\right.$ $\left.1 \mid k)] \leq \xi_{c}^{i}\right\}=p_{c}$.

The detailed proof of Theorem 3.1 is omitted here. The interested reader is referred to [9].

Remark 3.2: We usually cannot find the exact values of $\gamma_{p}^{i}$ and $\xi_{c}^{i}$, since the distribution function $F_{p}$ of $\Phi_{p}^{i-1} G_{p} w_{p}(k \mid k)+\ldots+G_{p} w_{p}(k+i-1 \mid k)$ involves a multivariate convolution integral with enormous computation for a long prediction horizon and a large dimensional system. Instead, we first discretize the probability density function of $w_{p, i}$ based on $q$ equi-spaced points on its domain. Then perform discrete scalar convolutions to compute a discrete approximation of the probability density function of $\Phi_{p}^{i-1} G_{p} w_{p}(k \mid k)+\ldots+$ $G_{p} w_{p}(k+i-1 \mid k)$. Finally the discrete approximation $\hat{F}_{p}$ to $F_{p}$ is calculated by using trapezoidal integration and further the values of $\gamma_{p}^{i}$ and $\xi_{c}^{i}$ can be approximated at reasonable computational cost. Note that the calculations of $\gamma_{p}^{i}$ and $\xi_{c}^{i}$ do not depend on the system state and can be performed offline.

To ensure recursive feasibility, define $T \mathbf{c}_{p}(k)=\left[c_{p}^{T}(k+\right.$ $\left.1 \mid k) \cdots c_{p}^{T}(k+N-1 \mid k) \mathbf{0}\right]^{T}$, where $T$ is the shift matrix with the identity matrix on its super-diagonal blocks and zeros elsewhere. Supposing that $\mathbf{c}_{p}(k)$ is a feasible solution at time $k$, the future feasibility at time $k+j, j=1,2, \ldots$, is then considered. Based on the information available at time $k+j$ and under the assumption that the vector of the perturbations at time $k+j$ is $\mathbf{c}_{p}(k+j)=T^{j} \mathbf{c}_{p}(k)$, the prediction of the system state at time $k+j+i$ is given as

$$
\begin{aligned}
& x_{p}(k+j+i \mid k+j) \\
= & \Phi_{p}^{i+j} z_{p}(k \mid k)+H_{p}^{i+j} \mathbf{c}_{p}(k)+\Phi_{p}^{i} e_{p}(k+j \mid k+j)+ \\
& \Phi_{p}^{i-1} G_{p} w_{p}(k+j \mid k+j)+\ldots+G_{p} w_{p}(k+j+i-1 \mid k+j)
\end{aligned}
$$

This provides the following predictions $y_{p}(k+j+i \mid k+j)$ and $\sum_{p=1}^{N_{p}} s_{c p}(k+j+i \mid k+j)$ based on information available at time $k$

$$
\begin{aligned}
& y_{p}(k+j+i \mid k+j) \\
= & \Psi_{p} \Phi_{p}^{i+j} z_{p}(k \mid k)+\left(\Psi_{p} H_{p}^{i+j}+D_{p} E_{p}^{i+j+1}\right) \mathbf{c}_{p}(k) \\
& +\Psi_{p} \Phi_{p}^{i} e_{p}(k+j \mid k+j)+\Psi_{p}\left[\Phi_{p}^{i-1} G_{p} w_{p}(k+j \mid k+j)\right. \\
& \left.+\ldots+G_{p} w_{p}(k+j+i-1 \mid k+j)\right]
\end{aligned}
$$

and

$$
\begin{aligned}
& \sum_{p=1}^{N_{p}} s_{c p}(k+j+i \mid k+j) \\
= & \sum_{p=1}^{N_{p}} \Delta_{c p} \Phi_{p}^{i+j} z_{p}(k \mid k)+\sum_{p=1}^{N_{p}}\left(\Delta_{c p} H_{p}^{i+j}+\right. \\
& \left.F_{c p} E_{p}^{i+j+1}\right) \mathbf{c}_{p}(k)+\sum_{p=1}^{N_{p}} \Delta_{c p} \Phi_{p}^{i} e_{p}(k+j \mid k+j)+ \\
& \sum_{p=1}^{N_{p}} \Delta_{c p}\left[\Phi_{p}^{i-1} G_{p} w_{p}(k+j \mid k+j)+\ldots+\right. \\
& \left.G_{p} w_{p}(k+j+i-1 \mid k+j)\right] .
\end{aligned}
$$


Note that $x_{p}(k+j+i \mid k+j)$ in (15) is expressed in terms of $e_{p}(k+j \mid k+j)$ rather than $e_{p}(k)$. This is because $e_{p}(k+j \mid k+j)$ depends on $w_{p}(k), w_{p}(k+1), \ldots, w_{p}(k+j-1)$, which, given that $x_{p}(k+j+i \mid k+j)$ is conditioned on information available at time $k+j$, cannot be considered to be random variables. Instead it is necessary to consider the worst-case value for $e_{p}(k+j \mid k+j)$ over the class of allowable additive uncertainty.

From (16)-(17), Theorem 3.1 can be modified by deriving the conditions for feasibility of $\mathbf{c}_{p}(k+j)=T^{j} \mathbf{c}_{p}(k)$ in (13)(14) at time $k+j$ for all $j \geq 1$.

Theorem 3.2: [9] Probabilistic constraints (4) and (6) are satisfied at time $k$, if $\mathbf{c}_{p}(k), p \in \mathcal{P}$, satisfies for $i=1,2, \ldots$,

$$
\begin{aligned}
& \Psi_{p} \Phi_{p}^{i} z_{p}(k \mid k)+\left(\Psi_{p} H_{p}^{i}+D_{p} E_{p}^{i+1}\right) \mathbf{c}_{p}(k) \\
\leq & h_{p}-\beta_{p}^{i}, \forall p \in \mathcal{P}, \\
& \sum_{p=1}^{N_{p}} \Delta_{c p} \Phi_{p}^{i} z_{p}(k \mid k)+\sum_{p=1}^{N_{p}}\left(\Delta_{c p} H_{p}^{i}+F_{c p} E_{p}^{i+1}\right) \mathbf{c}_{p}(k) \\
\leq & b_{c}-\zeta_{c}^{i}, \forall c \in \mathcal{C},
\end{aligned}
$$

where $\beta_{p}^{i}$ and $\zeta_{c}^{i}, i=1,2, \ldots$, are defined respectively as the maximum elements of the $i$ th columns of the matrices

$$
\left[\begin{array}{cccc}
\gamma_{p}^{1} & \gamma_{p}^{2} & \gamma_{p}^{3} & \cdots \\
0 & \gamma_{p}^{1}+a_{p}^{1} & \gamma_{p}^{2}+a_{p}^{2} & \cdots \\
0 & 0 & \gamma_{p}^{1}+a_{p}^{1}+a_{p}^{2} & \cdots \\
0 & 0 & 0 & \cdots \\
\vdots & \vdots & \vdots & \ddots
\end{array}\right]
$$

and

$$
\left[\begin{array}{cccc}
\xi_{c}^{1} & \xi_{c}^{2} & \xi_{c}^{3} & \cdots \\
0 & \xi_{c}^{1}+d_{c}^{1} & \xi_{c}^{2}+d_{c}^{2} & \cdots \\
0 & 0 & \xi_{c}^{1}+d_{c}^{1}+d_{c}^{2} & \cdots \\
0 & 0 & 0 & \cdots \\
\vdots & \vdots & \vdots & \ddots
\end{array}\right]
$$

with

$$
\begin{gathered}
a_{p}^{i}=\max _{w_{p} \in W_{p}} \Psi_{p} \Phi_{p}^{i} G_{p} w_{p}=\left|\Psi_{p} \Phi_{p}^{i} G_{p}\right| \alpha_{p}, \\
d_{c}^{i}=\max _{w_{p} \in W_{p}} \sum_{p=1}^{N_{p}} \Delta_{c p} \Phi_{p}^{i} G_{p} w_{p}=\sum_{p=1}^{N_{p}}\left|\Delta_{c p} \Phi_{p}^{i} G_{p}\right| \alpha_{p} .
\end{gathered}
$$

Furthermore, if conditions (18)-(19) are feasible at time $k$, then (18)-(19) will remain feasible at all times $k+j, j \geq 1$. The interested reader is referred to [9] for the detailed proof of Theorem 3.2. The CSMPC optimization problem $\mathbf{P}^{C}\left(x_{1}(k), \ldots, x_{N_{p}}(k)\right)$ is formulated as

$$
\min _{\mathbf{C}(k)} J_{k}(\mathbf{C}(k)) \text { in }(11)
$$

subject to for $i=1,2, \ldots$ :

$$
\begin{aligned}
& \Psi_{p} \Phi_{p}^{i} z_{p}(k \mid k)+\left(\Psi_{p} H_{p}^{i}+D_{p} E_{p}^{i+1}\right) \mathbf{c}_{p}(k) \\
& \leq h_{p}-\beta_{p}^{i}, \forall p \in \mathcal{P}, \\
& \sum_{p=1}^{N_{p}} \Delta_{c p} \Phi_{p}^{i} z_{p}(k \mid k)+\sum_{p=1}^{N_{p}}\left(\Delta_{c p} H_{p}^{i}+F_{c p} E_{p}^{i+1}\right) \mathbf{c}_{p}(k) \\
& \leq b_{c}-\zeta_{c}^{i}, \forall c \in \mathcal{C} .
\end{aligned}
$$

After the optimization problem is solved at each time step, the following input is applied to each subsystem $p \in \mathcal{P}$,

$$
u_{p}(k)=K_{p} x_{p}(k)+c_{p}(k \mid k) .
$$

Under this control, the CSMPC optimization problem is recursively feasible (due to Theorem 3.2) and the closed-loop system is stable (for details, see [22], Theorem 10) satisfying

$$
\begin{aligned}
\lim _{r \rightarrow \infty} \frac{1}{r+1} \sum_{k=0}^{r} \sum_{p=1}^{N_{p}} \mathbb{E} & x_{p}^{T}(k) Q x_{p}(k) \\
& \left.+u_{p}^{T}(k) R u_{p}(k)\right] \leq \sum_{p=1}^{N_{p}} L_{p} .
\end{aligned}
$$

To derive a DSMPC algorithm only involving a finite number of constraints in Section IV, we next show how to compute upper-bounds on $\beta_{p}^{i}$ and $\zeta_{c}^{i}$ which hold for all $i \geq 1$. These bounds also enable the constraint parameters in the online DSMPC optimization problem to be computed offline.

Define symmetric positive-definite matrix $S_{p} \in \mathbb{R}^{N_{x, p} \times N_{x, p}}$ and scalar $\rho_{p} \in(0,1)$ as the solution of the semi-definite program [22]

$$
\left(\rho_{p}, S_{p}\right)=\arg \min _{\substack{0<\rho_{p}<1 \\ S_{p}=S_{p}^{T} \succ 0}} \rho_{p}
$$

subject to

$$
\begin{gathered}
\Phi_{p} S_{p} \Phi_{p}^{T} \preceq \rho_{p}^{2} S_{p} \\
\left\|G_{p} w_{p}\right\|_{S_{p}^{-1}} \leq 1, \quad \forall w_{p} \in W_{p} .
\end{gathered}
$$

Note that the convexity of (27) and the strict stability of $\Phi_{p}$ ensure the existence and uniqueness of the optimal solution of (25)-(27).

Lemma 3.1: The sequences $\beta_{p}^{1}, \beta_{p}^{2}, \ldots$ and $\zeta_{c}^{1}, \zeta_{c}^{2}, \ldots$ are monotonically non-decreasing and converge to $\bar{\beta}_{p}$ and $\bar{\zeta}_{c}$ respectively, which are bounded by

$$
\begin{gathered}
\bar{\beta}_{p} \leq \gamma_{p}^{1}+\sum_{j=1}^{v_{p}-1} a_{p}^{j}+\frac{\rho_{p}^{v_{p}}}{1-\rho_{p}}\left\|\Psi_{p}^{T}\right\|_{S_{p}}, \forall p \in \mathcal{P}, \\
\bar{\zeta}_{c} \leq \xi_{c}^{1}+\sum_{i=1}^{\mu_{c}-1} d_{c}^{i}+\sum_{p=1}^{N_{p}} \frac{\rho_{p}^{\mu_{c}}}{1-\rho_{p}}\left\|\Delta_{c p}^{T}\right\|_{S_{p}}, \forall c \in \mathcal{C},
\end{gathered}
$$

for any integers $v_{p}>1$ and $\mu_{c}>1$.

Proof: The lemma is an extension of Corollary 4 in [9] to a case of multiple subsystems. In addition to local constraints, this paper also considers coupled constraints amongst several subsystems. Bounds on $\bar{\zeta}_{c}$ are derived so as to cater for coupled probabilistic constraints.

Remark 3.3: Provided that $v_{p}$ and $\mu_{c}$ are sufficiently large, the upper-bounds on $\bar{\beta}_{p}$ and $\bar{\zeta}_{c}$ in (28)-(29) can be made arbitrarily tight.

\section{DSMPC ALGORITHM}

To distribute $\mathbf{P}^{C}\left(x_{1}(k), \ldots, x_{N_{p}}(k)\right)$ amongst subsystems as local optimization problems, we first adopt the update strategy as in [17]. At each time $k$, only a single subsystem, 
defined as $p_{k}$, is permitted to optimize for a new plan, while each remaining subsystem uses the feasible candidate plan

$$
\begin{aligned}
\tilde{\mathbf{c}}_{q}(k) & \triangleq T \mathbf{c}_{q}^{*}(k-1) \\
& =\left[c_{q}^{* T}(k \mid k-1) \cdots c_{q}^{* T}(k+N-2 \mid k-1) \mathbf{0}\right]^{T}
\end{aligned}
$$

that is the tail of the previous solution $\mathbf{c}_{q}^{*}(k-1)$ augmented with 0 . The order in which subsystems' plans are optimized is determined by the update sequence $\left\{p_{1}, \ldots, p_{k}, p_{k+1}, \ldots\right\}$, which is chosen by the designer.

Then at time $k$, with the plans for subsystems $q$, $q \in \mathcal{Q}_{p_{k}}$, fixed by (30), the local optimization problem $\mathbf{P}_{p}^{D}\left(x_{p}(k) ; Z_{p}^{*}(k)\right)$ for optimizing subsystem $p=p_{k}$ is formulated as

$$
\begin{aligned}
\min _{\mathbf{c}_{p}(k)} J_{p}^{k}\left(\mathbf{c}_{p}(k)\right)= & \sum_{i=0}^{\infty} \mathbb{E}\left[x_{p}^{T}(k+i \mid k) Q x_{p}(k+i \mid k)\right. \\
& \left.+u_{p}^{T}(k+i \mid k) R u_{p}(k+i \mid k)-L_{p}\right]
\end{aligned}
$$

subject to (22) for subsystem $p$ only and the constraints, for $i=1,2, \ldots$,

$$
\begin{array}{r}
\Delta_{c p} \Phi_{p}^{i} z_{p}(k \mid k)+\Delta_{c p} H_{p}^{i} \mathbf{c}_{p}(k)+F_{c p} E_{p}^{i+1} \mathbf{c}_{p}(k)+ \\
\sum_{q \in \mathcal{P}_{c} \backslash\{p\}}\left[\Delta_{c q} \Phi_{q}^{i} z_{q}^{*}(k)+\left(\Delta_{c q} H_{q}^{i}+F_{c q} E_{q}^{i+1}\right) \mathbf{c}_{q}^{*}(k)\right] \\
\leq b_{c}-\zeta_{c}^{i}, \forall c \in \mathcal{C}_{p} .
\end{array}
$$

Note that the values of $\mathbf{c}_{q}(k)$ and $z_{q}(k), \forall q \in \mathcal{Q}_{p}$, are not affected by the decision variable $\mathbf{c}_{p}(k)$, so they appear as fixed values in (32), denoted by $*$.

Next, to ensure that (22) and (32) are satisfied over an infinite prediction horizon, a terminal constraint is designed below. Since $c_{p}(k+i \mid k)=\mathbf{0}$ for $i \geq N$, (22) and (32) for $i \geq N$ can be rewritten as

$$
\begin{aligned}
& \Psi_{p} \Phi_{p}^{j} z_{p}(k+N \mid k) \leq h_{p}-\beta_{p}^{N+j}, j=0,1,2, \ldots, \\
& \Delta_{c p} \Phi_{p}^{j} z_{p}(k+N \mid k) \leq b_{c}-\zeta_{c}^{N+j} \\
& \quad-\sum_{q \in \mathcal{P}_{c} \backslash\{p\}} \Delta_{c q} \Phi_{q}^{j} z_{q}^{*}(k+N \mid k), j=0,1,2, \ldots
\end{aligned}
$$

Following [23], define the maximal admissible set at time $k$ as $S_{p}^{\infty \mid k}$, which is the subset of $\mathbb{R}^{N_{x, p}}$ containing all $z_{p}(k+N \mid k)$ such that (33)-(34) hold for all $j \geq 0$. The upper-bounds on $\beta_{p}^{i}$ and $\zeta_{c}^{i}$ imply conditions for the existence of $S_{p}^{\infty \mid k}$.

Lemma 4.1: If the thresholds $l_{p}$ and $p_{c}$ are such that $h_{p}>\bar{\beta}_{p}$ and $b_{c}>\bar{\zeta}_{c}+\sum_{q \in \mathcal{P}_{c} \backslash\{p\}} \bar{b}_{c q}^{*}(k), \forall c \in \mathcal{C}_{p}$, with $\bar{b}_{c q}^{*}(k)=\max _{j=0,1, \ldots} \Delta_{c q} \Phi_{q}^{j} z_{q}^{*}(k+N \mid k)$, then the maximal admissible set $S_{p}^{\infty \mid k}$ is non-empty.

Proof: Since $\Phi_{q}$ is strictly stable by assumption, $z_{q}^{*}(k+$ $N+j \mid k)=\Phi_{q}^{j} z_{q}^{*}(k+N \mid k)$ converges to zero as $j \rightarrow \infty$ and $z_{q}^{*}(k+N+j \mid k)$ is uniformly bounded for any $k \geq 0$. Therefore the term $\Delta_{c q} \Phi_{q}^{j} z_{q}^{*}(k+N \mid k)$ remains bounded. Using $\bar{\beta}_{p}, \bar{\zeta}_{c}$, and $\bar{b}_{c q}^{*}(k)$ to respectively bound $\beta_{p}^{N+j}, \zeta_{c}^{N+j}$, and $\Delta_{c q} \Phi_{q}^{j} z_{q}^{*}(k+N \mid k)$ for $j=0,1, \ldots$ in (33)-(34), the conditions in Lemma 4.1 therefore ensure that $S_{p}^{\infty}{ }^{\infty k}$ contains the origin, which implies that $S_{p_{-}}^{\infty \mid k}$ is non-empty.

Because the exact values of $\bar{\beta}_{p}$ and $\bar{\zeta}_{c}$ are unknown, the conditions in Lemma 4.1 may be modified by using the bounds in (28)-(29)

$$
h_{p}>\gamma_{p}^{1}+\sum_{j=1}^{v_{p}-1} a_{p}^{j}+\frac{\rho_{p}^{v_{p}}}{1-\rho_{p}}\left\|\Psi_{p}^{T}\right\|_{S_{p}}
$$

and

$$
\begin{array}{r}
b_{c}>\xi_{c}^{1}+\sum_{i=1}^{\mu_{c}-1} d_{c}^{i}+\sum_{p=1}^{N_{p}} \frac{\rho_{p}^{\mu_{c}}}{1-\rho_{p}}\left\|\Delta_{c p}^{T}\right\|_{S_{p}}+ \\
\sum_{q \in \mathcal{P}_{c} \backslash\{p\}} \bar{b}_{c q}^{*}(k) .
\end{array}
$$

The result in [23] provides a method to compute an inner approximation of $S_{p}^{\infty \mid k}$. Replace $\beta_{p}^{N+j}$ and $\zeta_{c}^{N+j}$ by the bounds in (28)-(29) beyond a horizon $j=\hat{N}$ and define the terminal set $S_{p}^{\hat{N} \mid k}$ as follows

$$
\begin{aligned}
& S_{p}^{\hat{N \mid}} \triangleq\left\{z \mid \Psi_{p} \Phi_{p}^{j} z \leq h_{p}-\beta_{p}^{N+j}, \Delta_{c p} \Phi_{p}^{j} z \leq b_{c}-\right. \\
& \zeta_{c}^{N+j}-\sum_{q \in \mathcal{P}_{c} \backslash\{p\}} \Delta_{c q} \Phi_{q}^{j} z_{q}^{*}(k+N \mid k), \\
& \forall c \in \mathcal{C}_{p}, \quad j=0,1, \ldots, \hat{N} ; \\
& \Psi_{p} \Phi_{p}^{j} z \leq h_{p}-\gamma_{p}^{1}-\sum_{j=1}^{v_{p}-1} a_{p}^{j}-\frac{\rho_{p}^{v_{p}}}{1-\rho_{p}}\left\|\Psi_{p}^{T}\right\|_{S_{p}}, \\
& \Delta_{c p} \Phi_{p}^{j} z+\sum_{q \in \mathcal{P}_{c} \backslash\{p\}} \Delta_{c q} \Phi_{q}^{j} z_{q}^{*}(k+N \mid k) \leq b_{c}-\xi_{c}^{1}- \\
& \sum_{c}-1 \\
& \left.\sum_{i=1}^{N_{p}} d_{c}^{i}-\sum_{p=1}^{N_{p}} \frac{\rho_{p}^{\mu_{c}}}{1-\rho_{p}}\left\|\Delta_{c p}^{T}\right\|_{S_{p}}, \forall c \in \mathcal{C}_{p}, j>\hat{N}\right\} .(37)
\end{aligned}
$$

The inner approximation can be made arbitrarily tight to $S_{p}^{\infty \mid k}$ by using a sufficiently large value of $\hat{N}$. Furthermore, it can be shown that there exists an integer $n^{*}$ such that $S_{p}^{\hat{N} \mid k}$ is in fact determined by only the first $\hat{N}+n^{*}$ inequalities, i.e., the constraints from $j=\hat{N}+n^{*}+1$ to $j=\infty$ can be removed without effect, whenever $S_{p}^{\infty \mid k}$ is bounded. We denote the terminal set which involves a finite number of inequalities by $S_{p}^{\hat{N}, n^{*} \mid k}$. The smallest allowable value of $n^{*}$ can be determined by solving a finite number of linear programming problems (for details, see [23]).

A revised form of $\mathbf{P}_{p}^{D}\left(x_{p}(k) ; Z_{p}^{*}(k)\right)$ is now obtained

$$
\min _{\mathbf{c}_{p}(k)} J_{p}^{k}\left(\mathbf{c}_{p}(k)\right) \text { in }(31)
$$

subject to (22) for subsystem $p$ and (32) for $i=1,2, \ldots, N-$ 1 , and the terminal constraint $z_{p}(k+N \mid k) \in S_{p}^{\hat{N}, n^{*} \mid k}$.

Remark 4.1: Given the distribution of $w_{p}$, the cost function $J_{p}^{k}\left(\mathbf{c}_{p}(k)\right)$ in (31) can be written as a quadratic function of $\mathbf{c}_{p}(k)$ (see [22], Remark 9). In addition, (22) and (32) are affine constraint functions of $\mathbf{c}_{p}(k)$ and $S_{p}^{\hat{N}, n^{*} \mid k}$ is a polyhedron with $\mathbf{c}_{p}(k)$. The problem $\mathbf{P}_{p}^{D}\left(x_{p}(k) ; Z_{p}^{*}(k)\right)$ can therefore be cast as a quadratic program $(\mathrm{QP})$, which can be solved efficiently.

In optimization problem $\mathbf{P}_{p}^{D}\left(x_{p}(k) ; Z_{p}^{*}(k)\right), Z_{p}^{*}(k)$ denotes the collection of information about other subsystems' states $z_{q}^{*}(k)$ and the vectors of perturbations $\mathbf{c}_{q}^{*}(k)$ required by $p$ to evaluate constraint (32). It can be seen that to evaluate (32), 
subsystem $p$ must have knowledge of $\mathbf{c}_{q}^{*}(k)$ for each subsystem $q \in \mathcal{Q}_{p}$. Denote by $\hat{k}_{q}$ the last step, before the current step, at which subsystem $q$ is updated [17]

$$
\hat{k}_{q} \triangleq \max _{k^{\prime} \in\left\{k^{\prime}<k \mid p_{k^{\prime}}=q\right\}} k^{\prime} .
$$

Then the vectors of perturbations $\mathbf{c}_{q}^{*}(k)$ appearing in (32) can be expressed as $\mathbf{c}_{q}^{*}(k)=T^{k-\hat{k}_{q}} \mathbf{c}_{q}^{*}\left(\hat{k}_{q}\right), \forall q \in \mathcal{Q}_{p}$, for all $k \leq$ $\hat{k}_{q}+N-1$. For greater values of $k, \mathbf{c}_{q}^{*}(k)=\mathbf{0}$. Hence, one strategy to achieve the information requirements for $Z_{p}^{*}(k)$ is provided as follows. At each time $k$, all subsystems in $\mathcal{Q}_{p}$ sample the current states and transmit them to the optimizing subsystem $p$. After solving $\mathbf{P}_{p}^{D}\left(x_{p}(k) ; Z_{p}^{*}(k)\right)$, the new plan of the optimizing subsystem $p$ must be communicated to all subsystems $q \in \mathcal{Q}_{p}$. This strategy is adopted in the following algorithm, which is executed by all subsystems in parallel.

Algorithm 1 (DSMPC Algorithm):

Offline. Determine $v_{p}, \mu_{c}$, and horizons $\hat{N}$ and $n^{*}$. Compute $\beta_{p}^{i}$ and $\zeta_{c}^{i}, i=1,2, \ldots, N+\hat{N}$, in (20)-(21). Compute $\gamma_{p}^{1}$, $\xi_{c}^{1}, a_{p}^{j}, j=1,2, \ldots, v_{p}-1$, and $d_{c}^{i}, i=1,2, \ldots, \mu_{c}-1$, to complete the definition of $S_{p}^{\hat{N}, n^{*} \mid k}$.

\section{Online.}

(i) Initialization: Wait for a feasible solution $\mathbf{c}_{p}^{*}(0)$ and information $Z_{p}^{*}(0)$ from the central initializing controller for $\forall p \in \mathcal{P}$. If a solution cannot be found, stop.

(ii) All subsystems $p$ : Apply $u_{p}^{*}(k)=K_{p} x_{p}(k)+c_{p}^{*}(k)$.

(iii) All subsystems $p$ : Update $k$ and sample $x_{p}(k)$.

Subsystems $q \in \mathcal{Q}_{p_{k}}$ : Transmit $x_{q}(k)$ to subsystem $p_{k}$.

(iv) Update plan:

\section{Subsystem $p=p_{k}$ :}

a) Solve $\mathbf{P}_{p}^{D}\left(x_{p}(k) ; Z_{p}^{*}(k)\right)$ to obtain new plan $\mathbf{c}_{p}^{*}(k)$.

b) Transmit the new plan to subsystems $q, q \in \mathcal{Q}_{p}$.

c) Set $\hat{k}_{p}=k$.

Subsystems $p \neq p_{k}$ : Construct new plans $\mathbf{c}_{p}^{*}(k)=$ $T^{k-\hat{k}_{p}} \mathbf{c}_{p}^{*}\left(\hat{k}_{p}\right)$ with zero update.

(v) Go to step (ii).

The following theorem states the main result of this paper.

Theorem 4.1: If there exists a feasible (but not necessarily optimal) solution $\mathbf{c}_{p}^{*}(0)=$ $\left[c_{p}^{* T}(0 \mid 0) \quad c_{p}^{* T}(1 \mid 0) \cdots c_{p}^{* T}(N-1 \mid 0)\right]^{T}, p \in \mathcal{P}$, to the optimization problem $\mathbf{P}^{C}\left(x_{1}(0), \ldots, x_{N_{p}}(0)\right)$ at time $k=0$, and the system is controlled according to Algorithm 1, then all subsequent optimization problems $\mathbf{P}_{p}^{D}\left(x_{p}(k) ; Z_{p}^{*}(k)\right)$ are feasible for $k>0$. Furthermore, for any choice of update sequence, the closed-loop stability of the entire system is guaranteed, which satisfies

$$
\begin{aligned}
\lim _{r \rightarrow \infty} \frac{1}{r+1} \sum_{k=0}^{r} \sum_{p=1}^{N_{p}} \mathbb{E}\left[x_{p}^{T}(k \mid k) Q x_{p}(k \mid k)\right. & + \\
& \left.u_{p}^{T}(k \mid k) R u_{p}(k \mid k)\right] \leq \sum_{p=1}^{N_{p}} L_{p} .
\end{aligned}
$$

Proof: From the conditions of Theorem 3.2, if there exists initial feasible solutions $\left\{\mathbf{c}_{p}^{*}(0)\right\}_{p \in \mathcal{P}}$ to the problem $\mathbf{P}^{C}\left(x_{1}(0), \ldots, x_{N_{p}}(0)\right),\left\{T \mathbf{c}_{p}^{*}(0)\right\}_{p \in \mathcal{P}}$ are feasible solutions to $\mathbf{P}^{C}\left(x_{1}(1), \ldots, x_{N_{p}}(1)\right)$. It is easy to see that, at time 1 , for optimizing subsystem $p_{1}, T \mathbf{c}_{p_{1}}^{*}(0)$ directly satisfies (22). Meanwhile, by choosing $z_{q}^{*}(1)=x_{q}(1), \mathbf{c}_{q}^{*}(1)=T \mathbf{c}_{q}^{*}(0)$, $z_{p_{1}}(1)=x_{p_{1}}(1)$, and $\mathbf{c}_{p_{1}}(1)=T \mathbf{c}_{p_{1}}^{*}(0)$, (32) is equivalent to (23) when $\mathbf{P}^{C}\left(x_{1}(1), \ldots, x_{N_{p}}(1)\right)$ adopts $\left\{T \mathbf{c}_{p}^{*}(0)\right\}_{p \in \mathcal{P}}$, then $T \mathbf{c}_{p_{1}}^{*}(0)$ satisfies (32). Hence, $T \mathbf{c}_{p_{1}}^{*}(0)$ is a feasible solution to $\mathbf{P}_{p_{1}}^{D}\left(x_{p_{1}}(1) ; Z_{p_{1}}^{*}(1)\right)$.

Next, we assume that feasible solutions $\left\{\mathbf{c}_{p}^{*}(k-\right.$ 1) $\}_{p \in \mathcal{P}}$ are available at time $k-1$. By Algorithm 1, at time $k$, all subsystems $q \neq p_{k}$ renew their plan$\mathrm{s}$ to be $\left\{T \mathbf{c}_{q}^{*}(k-1)\right\}_{q \neq p_{k}}$. For optimizing subsystem $p_{k}$, solving $\mathbf{P}_{p_{k}}^{D}\left(x_{p_{k}}(k) ; Z_{p_{k}}^{*}(k)\right)$ is equivalent to solving $\mathbf{P}^{C}\left(x_{1}(k), \ldots, x_{N_{p}}(k)\right)$ constrained to take $\mathbf{c}_{q}^{*}(k)=T \mathbf{c}_{q}^{*}(k-$ 1) for $q \neq p_{k}$. From recursive feasibility of CSMPC optimization problem in Theorem 3.2, $\left\{T \mathbf{c}_{p}^{*}(k-1)\right\}_{p \in \mathcal{P}}$ are feasible for $\mathbf{P}^{C}\left(x_{1}(k), \ldots, x_{N_{p}}(k)\right)$, and $T \mathbf{c}_{p_{k}}^{*}(k-1)$ is also feasible for $\mathbf{P}_{p_{k}}^{D}\left(x_{p_{k}}(k) ; Z_{p_{k}}^{*}(k)\right)$. By recursion, a feasible solution to $\mathbf{P}^{C}\left(x_{1}(0), \ldots, x_{N_{p}}(0)\right)$ implies that all subsequent optimization problems $\mathbf{P}_{p_{k}}^{D}\left(x_{p_{k}}(k) ; Z_{p_{k}}^{*}(k)\right), k>0$, are feasible regardless of the choice of update sequence.

To consider closed-loop stability, the global cost is defined as the summation of the local cost of each subsystem $\mathcal{J}(k) \triangleq \sum_{p=1}^{N_{p}} J_{p}^{k}\left(\mathbf{c}_{p}(k)\right)$. Assume that $\left\{\mathbf{c}_{p}^{*}(k)\right\}_{p \in \mathcal{P}}$ are feasible solutions at time $k$ and the corresponding local cost is $J_{p}^{k}\left(\mathbf{c}_{p}^{*}(k)\right)$ for subsystem $p \in \mathcal{P}$. By Algorithm 1, at time $k+1$, the optimizing subsystem $p_{k+1}$ obtains its solution $\mathbf{c}_{p_{k+1}}^{*}(k+1)$ by solving $\mathbf{P}_{p_{k+1}}^{D}\left(x_{p_{k+1}}(k+1) ; Z_{p_{k+1}}^{*}(k+1)\right)$, while all other subsystems $q \neq p_{k+1}$ adopt their respective candidate solutions $\mathbf{c}_{q}^{*}(k+1)=T \mathbf{c}_{q}^{*}(k)$. Thus, the global cost at time $k+1$ is given as

$$
\mathcal{J}^{*}(k+1) \triangleq J_{p_{k+1}}^{k+1}\left(\mathbf{c}_{p_{k+1}}^{*}(k+1)\right)+\sum_{q \neq p_{k+1}} J_{q}^{k+1}\left(T \mathbf{c}_{q}^{*}(k)\right) .
$$

Taking expectation on both sides of (39), it follows that

$$
\begin{gathered}
\mathbb{E}\left[\mathcal{J}^{*}(k+1)\right]=\mathbb{E}[\tilde{\mathcal{J}}(k+1)]+\mathbb{E}\left[J_{p_{k+1}}^{k+1}\left(\mathbf{c}_{p_{k+1}}^{*}(k+1)\right)-\right. \\
\left.J_{p_{k+1}}^{k+1}\left(T \mathbf{c}_{p_{k+1}}^{*}(k)\right)\right],
\end{gathered}
$$

where $\tilde{\mathcal{J}}(k+1)$ is the global cost associated with each subsystem adopting their respective candidate plans $T \mathbf{c}_{p}^{*}(k)$. Since $T \mathbf{c}_{p_{k+1}}^{*}(k)$ is a known feasible solution at time $k+1$ for subsystem $p_{k+1}$, the cost associated with $T \mathbf{c}_{p_{k+1}}^{*}(k)$ is an upper-bound on the optimal cost $J_{p_{k+1}}^{k+1}\left(\mathbf{c}_{p_{k+1}}^{*}(k+1)\right) \leq$ $J_{p_{k+1}}^{k+1}\left(T \mathbf{c}_{p_{k+1}}^{*}(k)\right)$. The last term of (40) is therefore less than or equal to zero, leaving

$$
\mathbb{E}\left[\mathcal{J}^{*}(k+1)\right] \leq \mathbb{E}[\tilde{\mathcal{J}}(k+1)] .
$$

Since $T \mathbf{c}_{p}^{*}(k)$ is a feasible solution at time $k+1$, so that

$$
\begin{aligned}
J_{p}^{k}\left(\mathbf{c}_{p}^{*}(k)\right)= & \mathbb{E}\left[J_{p}^{k+1}\left(T \mathbf{c}_{p}^{*}(k)\right)\right]+x_{p}^{T}(k) Q x_{p}(k) \\
& +u_{p}^{T}(k) R u_{p}(k)-L_{p} .
\end{aligned}
$$

Summing (42) over $p=1,2, \ldots, N_{p}$ and taking expectation on both sides, we obtain

$$
\begin{aligned}
\mathbb{E}\left[\mathcal{J}^{*}(k)\right]= & \mathbb{E}[\tilde{\mathcal{J}}(k+1)]+\sum_{p=1}^{N_{p}} \mathbb{E}\left[x_{p}^{T}(k) Q x_{p}(k)+\right. \\
& \left.u_{p}^{T}(k) R u_{p}(k)-L_{p}\right] .
\end{aligned}
$$


Following the above equality, (41) is equivalent to

$$
\begin{aligned}
\mathbb{E}\left[\mathcal{J}^{*}(k+1)\right] \leq & \mathbb{E}\left[\mathcal{J}^{*}(k)\right]-\sum_{p=1}^{N_{p}} \mathbb{E}\left[x_{p}^{T}(k) Q x_{p}(k)+\right. \\
& \left.u_{p}^{T}(k) R u_{p}(k)-L_{p}\right] .
\end{aligned}
$$

Summing (44) over $k=0,1, \ldots, r$ leads to

$$
\begin{array}{r}
\sum_{k=0}^{r} \sum_{p=1}^{N_{p}} \mathbb{E}\left[x_{p}^{T}(k) Q x_{p}(k)+u_{p}^{T}(k) R u_{p}(k)-L_{p}\right] \leq \\
\mathbb{E}\left[\mathcal{J}^{*}(0)\right]-\mathbb{E}\left[\mathcal{J}^{*}(r+1)\right] .
\end{array}
$$

Because of the finite $\mathcal{J}^{*}(0)$ by assumption and the lower boundedness of $\mathbb{E}\left[\mathcal{J}^{*}(r+1)\right]$ by the quadratic form of $J_{p}^{k}\left(\mathbf{c}_{p}^{*}(k)\right.$ ) (see Remark 4.1), (38) can be implied by (45), which completes the proof.

\section{Numerical EXAMPLES}

\section{A. Example 1: Homogeneous subsystems}

Consider a set of three identical subsystems, of which the model parameters are given as follows (see [8] and [24])

$$
\begin{gathered}
A_{p}=\left[\begin{array}{cc}
1 & 0.0075 \\
-0.143 & 0.996
\end{array}\right], B_{p}=\left[\begin{array}{l}
4.798 \\
0.115
\end{array}\right], \\
G_{p}=\left[\begin{array}{ll}
1 & 0 \\
0 & 1
\end{array}\right], \forall p \in \mathcal{P}=\{1,2,3\} .
\end{gathered}
$$

There are four probabilistic constraints with

$$
\begin{gathered}
C_{p}=\left[\begin{array}{ll}
1 & 0
\end{array}\right], D_{p}=0, h_{p}=2, l_{p}=0.8, \\
E_{c p}=\left[\begin{array}{cc}
1 & 0
\end{array}\right], F_{c p}=0, b_{c}=5.5, p_{c}=0.8, \\
\forall p \in \mathcal{P}, \forall c \in \mathcal{C}=\{1\} .
\end{gathered}
$$

Each element $w_{p, i}(k)$ is truncated from a Gaussian distribution with mean zero and variance $\frac{1}{25^{2}}$ and satisfies $\left|w_{p, i}(k)\right| \leq$ 0.075 for $i=1,2$. The feedback gain $K_{p}$ is taken as $K_{p}=$ $-[0.286-0.491]$, which is the LQ-optimal gain for the given plant model. The prediction horizons $N$ and $\hat{N}$ over which constraints are handled explicitly are chosen as $N=8$ and $\hat{N}=9$. For the chosen $N$ and $\hat{N}$, the set $S_{p}^{\hat{N}, n^{*} \mid k}$ is given with $n^{*}=1$. The update sequence employed is $\{1,2,3,1,2,3, \ldots\}$. The weights in the cost are $Q=\operatorname{diag}\{1,10\}$ and $R=1$.

By adopting Algorithm 1 and the unconstrained optimal control, we carry out 5000 Monte Carlo simulations, starting from the same initial conditions $x_{1}(0)=\left[\begin{array}{lll}3.5 & 3\end{array}\right]^{T}, x_{2}(0)=$ $\left[\begin{array}{ll}2.5 & 2\end{array}\right]^{T}$, and $x_{3}(0)=\left[\begin{array}{ll}2.5 & 2.8\end{array}\right]^{T}$. Fig. 1 and Fig. 2 show the closed-loop trajectories $\left\{x_{p}(k), k=0,1, \ldots\right\}$ for each subsystem and the sum of states $\left\{\sum_{p=1}^{3} x_{p}(k), k=0,1, \ldots\right\}$.

With the unconstrained optimal control, the local probabilistic constraints are violated at time $k \leq 3$ for every realization of the uncertainty sequence. For the trajectories obtained under Algorithm 1, 19.92\%, 0\%, and 20.17\% of the same 5000 realizations violate the local constraints for subsystems 1, 2 , and 3 at time $k=1,20.06 \%, 0 \%$, and $1.09 \%$ at time $k=2$, and $0.92 \%, 19.95 \%$, and $0.06 \%$ at time $k=3$. By Algorithm 1, the observed probability of violating the coupled state constraint is $19.95 \%$ at time $k=1,0.04 \%$ at time $k=2$, and $7.06 \%$ at time $k=3$, whereas violation rate is $100 \%$ at time $k \leq 3$ under the unconstrained optimal control.

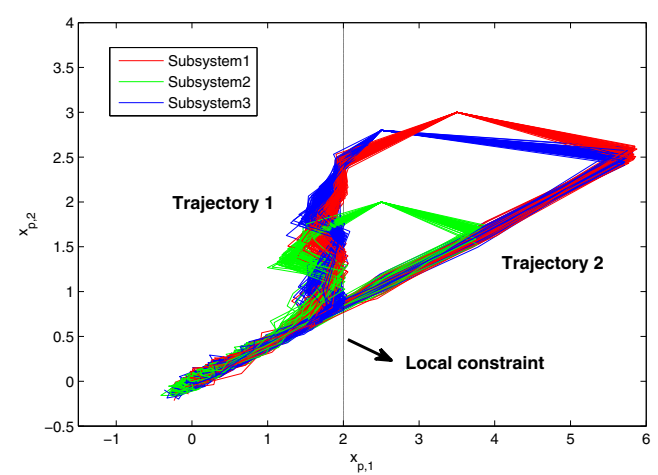

Fig. 1: Closed-loop trajectories of homogeneous subsystems under Algorithm 1 (Trajectory 1) and unconstrained optimal control (Trajectory 2) for 100 realizations of the uncertainty sequence.

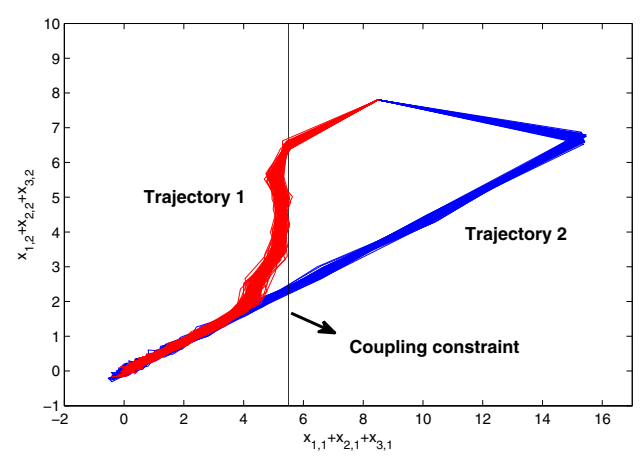

Fig. 2: Evolution of the sum of states $\left\{\sum_{p=1}^{3} x_{p}(k), k=0,1, \ldots\right\}$ when the system is controlled by Algorithm 1 (Trajectory 1) and unconstrained optimal control (Trajectory 2) for 100 realizations of the uncertainty sequence.

Moreover, the performance of Algorithm 1 is also compared with that of the unconstrained optimal control. The performance measured in this instance is the weighted sum of local costs over the duration of simulation. Algorithm 1 produces the cumulative cost (three subsystems, 15 samples) for closed-loop operation averaged over 5000 simulations of 558.06 , as compared with the average cost of 410.20 for the unconstrained optimal control. As expected, the unconstrained optimal control achieves a lower value of the average cost at the expense of violating the probabilistic constraints.

\section{B. Example 2: Heterogeneous subsystems}

Consider three different subsystems, which are modeled by

$$
\begin{aligned}
& A_{1}=\left[\begin{array}{cc}
1.6 & 1.1 \\
-0.7 & 1.2
\end{array}\right], B_{1}=\left[\begin{array}{l}
1 \\
1
\end{array}\right], \\
& A_{2}=\left[\begin{array}{cc}
1.5 & 1.1 \\
0 & 1.2
\end{array}\right], B_{2}=\left[\begin{array}{l}
0.8 \\
0.9
\end{array}\right], \\
& A_{3}=\left[\begin{array}{cc}
1.4 & 1.2 \\
-0.3 & 1.1
\end{array}\right], B_{3}=\left[\begin{array}{l}
1.2 \\
0.8
\end{array}\right], \\
& G_{p}=\left[\begin{array}{ll}
1 & 0 \\
0 & 1
\end{array}\right], \forall p \in \mathcal{P}=\{1,2,3\} .
\end{aligned}
$$

Each element $w_{p, i}(k)$ is truncated from a Gaussian distribution with mean zero and variance $\frac{1}{12^{2}}$ such that $\left|w_{p, i}(k)\right| \leq 0.5$, 
$i=1,2$. Let the update sequence be $\{1,2,3,1,2,3, \ldots\}, N=$ $6, \hat{N}=7, Q=I$, and $R=1$. The constraint parameters are

$$
\begin{gathered}
C_{1}=\left[\begin{array}{cc}
1 & 1.3
\end{array}\right], C_{2}=\left[\begin{array}{ll}
1.4 & 0.6
\end{array}\right], C_{3}=\left[\begin{array}{ll}
0.9 & 0.4
\end{array}\right], \\
D_{p}=0, l_{p}=0.8, h_{1}=15, h_{2}=8.4, h_{3}=9, \\
E_{c p}=C_{p}, F_{c p}=0, b_{c}=33, p_{c}=0.8, \forall p \in \mathcal{P} .
\end{gathered}
$$

In Fig. 3, a comparison result between Algorithm 1 and the unconstrained optimal control is shown for three different subsystems with initial conditions $x_{1}(0)=\left[\begin{array}{ll}-6 & 25\end{array}\right]^{T}$, $x_{2}(0)=[-345]^{T}$, and $x_{3}(0)=[-660]^{T}$. Under Algorithm 1, for 5000 realizations of the uncertainty sequence, the observed probabilities of violating the local constraints are $20.72 \%$, $20.24 \%$, and $19.50 \%$ for subsystems 1,2 , and 3 at time $k=1$, respectively, whereas for the unconstrained optimal control, the trajectories suggest that the violation rate is $100 \%$ at time $k=1$. From the simulation results, under Algorithm 1, the system states always converge to a neighborhood of the origin with the guarantee of constraint satisfaction despite the action of unknown but bounded disturbances.

Compare the performances of DSMPC and DRMPC (assuming $l_{p}=1$ and $p_{c}=1$ ) in terms of the achieved costs. The cumulative costs (three subsystems, eight samples) for closed-loop operation averaged over 5000 simulations are 89.63 and 125.71 under DSMPC and DRMPC respectively. As expected, the proposed DSMPC algorithm achieves an average improvement in the closed-loop performance of $40.24 \%$ with respect to DRMPC by allowing constraint violations to occur.

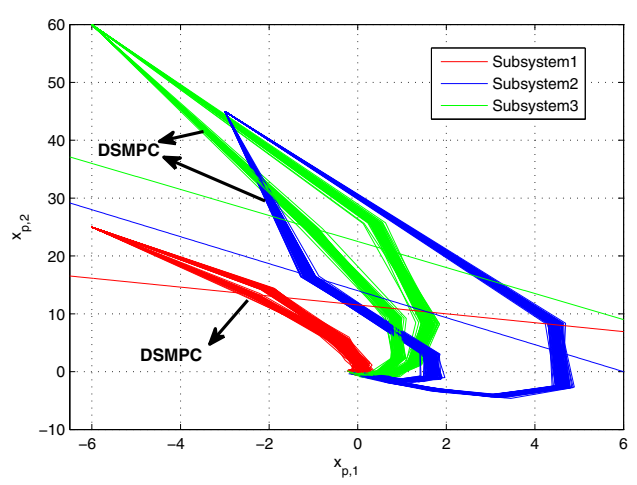

Fig. 3: Closed-loop trajectories of heterogeneous subsystems under Algorith$\mathrm{m} 1$ and unconstrained optimal control for 100 realizations of the uncertainty sequence.

\section{CONCLUSION}

In this paper, a DSMPC algorithm is proposed for a team of linear stochastic subsystems with additive uncertainty and probabilistic constraints. Coupled probabilistic constraints are handled non-conservatively in a distributed way. Recursive feasibility is guaranteed with respect to both local and coupled probabilistic constraints. The closed-loop stability of the whole system is ensured in the presence of stochastic disturbances.

On-going research is developing a DSMPC strategy for a group of stochastic subsystems with both additive and multiplicative uncertainty. One possible future direction is to account for the event-based MPC for uncertain systems and further promote it to the distributed case.

\section{REFERENCES}

[1] D. H. Van Hessem and O. H. Bosgra, "A conic reformulation of model predictive control including bounded and stochastic disturbances under state and input constraints," in Proc. 41st IEEE Conf. Decision Control, 2002, vol. 4, pp. 4643-4648.

[2] B. Kouvaritakis, M. Cannon, and V. Tsachouridis, "Recent developments in stochastic MPC and sustainable development," Annu. Rev. Control, vol. 28, no. 1, pp. 23-35, 2004.

[3] J. A. Primbs, "A soft constraint approach to stochastic receding horizon control," in Proc. 46th IEEE Conf. Decision Control, 2007, pp. 47974802.

[4] A. Mesbah, "Stochastic model predictive control: An overview and perspectives for future research," IEEE Contr. Syst., Accepted.

[5] J. Liu, D. M. de la Peña, and P. D. Christofides, "Distributed model predictive control of nonlinear systems subject to asynchronous and delayed measurements," Automatica, vol. 46, no. 1, pp. 52-61, 2010.

[6] B. E. Ydstie, "New vistas for process control: integrating physics and communication networks," AIChE J., vol. 48, no. 3, pp. 422-426, 2002.

[7] Y. Kuwata, A. Richards, T. Schouwenaars, and J. P. How, "Distributed robust receding horizon control for multivehicle guidance," IEEE Trans. Control Syst. Technol., vol. 15, no. 4, pp. 627-641, 2007.

[8] M. Cannon, B. Kouvaritakis, S. V. Raković, and Q. Cheng, "Stochastic tubes in model predictive control with probabilistic constraints," IEEE Trans. Autom. Control, vol. 56, no. 1, pp. 194-200, 2011.

[9] B. Kouvaritakis, M. Cannon, S. V. Raković, and Q. Cheng, "Explicit use of probabilistic distributions in linear predictive control," Automatica, vol. 46, no. 10, pp. 1719-1724, 2010.

[10] P. Hokayem, E. Cinquemani, D. Chatterjee, F. Ramponi, and J. Lygeros, "Stochastic receding horizon control with output feedback and bounded controls," Automatica, vol. 48, no. 1, pp. 77-88, 2012.

[11] J. A. Paulson, S. Streif, and A. Mesbah, "Stability for receding-horizon stochastic model predictive control," in Proc. 34th American Control Conf., 2015, pp. 937-943.

[12] L. Blackmore, M. Ono, A. Bektassov, and B. C. Williams, "A probabilistic particle-control approximation of chance-constrained stochastic predictive control," IEEE Trans. on Robotics, vol. 26, no. 3, pp. 502C517, 2010.

[13] G. C. Calafiore and L. Fagiano, "Stochastic model predictive control of LPV systems via scenario optimization," Automatica, vol. 49, no. 6, pp. 1861-1866, 2013.

[14] S. L. Waslander, G. Inalhan, and C. J. Tomlin, "Decentralized optimization via Nash bargaining," in Theory and Algorithms for Cooperative Systems. Singapore: World Scientific, 2004.

[15] T. Keviczky, F. Borrelli, and G. J. Balas, "Decentralized receding horizon control for large scale dynamically decoupled systems," Automatica, vol. 42, no. 12, pp. 2105-2115, 2006.

[16] A. Richards and J. P. How, "Robust distributed model predictive control," Int. J. Control, vol. 80, no. 9, pp. 1517-1531, 2007.

[17] P. Trodden and A. Richards, "Distributed model predictive control of linear systems with persistent disturbances," Int. J. Control, vol. 83, no. 8, pp. 1653-1663, 2010.

[18] A. Richards and J. How, "A decentralized algorithm for robust constrained model predictive control," in Proc. 23th American Control Conf., 2004, vol. 5, pp. 4261-4266.

[19] D. Lyons, J. Calliess, and U. D. Hanebeck, "Chance constrained model predictive control for multi-agent systems with coupling constraints," in Proc. 31st American Control Conf., 2012, pp. 1223-1230.

[20] L. Dai, Y. Xia, Y. Gao, B. Kouvaritakis, and M. Cannon, "Cooperative distributed stochastic MPC for systems with state estimation and coupled probabilistic constraints," Automatica, vol. 61, pp. 89-96, 2015.

[21] M. Cannon, B. Kouvaritakis, and X. Wu, "Probabilistic constrained MPC for multiplicative and additive stochastic uncertainty," IEEE Trans. Autom. Control, vol. 54, no. 7, pp. 1626-1632, 2009.

[22] M. Cannon, Q. Cheng, B. Kouvaritakis, and S. V. Raković, "Stochastic tube MPC with state estimation," Automatica, vol. 48, no. 3, pp. 536-541, 2012.

[23] E. G. Gilbert and K. T. Tan, "Linear systems with state and control constraints: the theory and practice of maximal admissible sets," IEEE Trans. Autom. Control, vol. 36, no. 9, pp. 1008-1020, 1991.

[24] M. Lazar, W. Heemels, B. J. P. Roset, H. Nijmeijer, and P. P. J. van den Bosch, "Input-to-state stabilizing sub-optimal NMPC with an application to DC-DC converters," Int. J. Robust Nonlin. Control, vol. 18, no. 8, pp. 890-904, 2008 